American Journal of Agricultural and Biological Sciences 3 (3): 575-578, 2008

ISSN 1557-4989

(C) 2008 Science Publications

\title{
An Unusual Association between Chromoblastomycosis and Jorge Lobo's Disease in the Same Patient from the Amazon Region
}

\author{
${ }^{1}$ Maurimélia Mesquita da Costa, ${ }^{1}$ Silvia H. Marques da Silva, ${ }^{1}$ Hugo J. R. Almeida, \\ ${ }^{1}$ Francisco Lúzio de P. Ramos, ${ }^{2}$ Maysa de Vasconcelos Brito ${ }^{2}$ Deborah Unger, ${ }^{2}$ Clívia Oliveira,

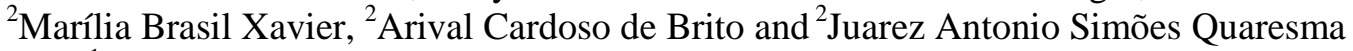 \\ ${ }^{1}$ Evandro Chagas Institute, SVS, Ministry of Health, Ananindeua, PA, Brazil \\ ${ }^{2}$ Tropical Medicine Unit, Federal do Para University, Belem, PA, Brazil
}

\begin{abstract}
A clinical case of co-infection Cladophialophora sp. and Lacazia loboi is described and in a same patient with an 18-year history of verrucous plaques and parakeloidal nodular lesions. A 50-yearold man, a laborer, living in the Anajás city, Para, Brazil (rural Amazon region), was seen at the Evandro Chagas Institute's ambulatory. Histopathological analysis revealed intense hyperplasia and hyperkeratosis of the epidermis, sometimes showing well-circumscribed areas of an intraepidermal suppurative content intermingled with round and brownish structures. The papillary and reticular dermis was characterized by fibrosis and a granulomatous reaction consisting of lymphocytes, histiocytes and giant cells permeated by suppurative inflammatory foci and multiple round fungal structures mainly arranged amidst a neutrophilic inflammatory infiltrate. The clinical material collected from the lesion was submitted to direct mycological examination using $20 \% \mathrm{KOH}$ solution and lactophenol cotton blue, which revealed the presence of yeast-like cells with a double membrane arranged in groups, a characteristic of Lacazia loboi, as well as sclerotic cells characteristic of chromoblastomycosis. Culture of the specimen on Mycosel agar and microculture on potato agar showed the typical morphology of Cladophialophora sp. The association of these two fungi causing lesions and inducing long-term disease may indicate a similarity in the characteristics of the habitat of these agents in the Amazon region.
\end{abstract}

Key words: Mycoses, fungus, Amazon, lacaziosis

\section{INTRODUCTION}

Subcutaneous mycoses generally result from the implantation of certain pathogenic fungi in the skin or subcutaneous tissue, which is frequently due to trauma. These mycoses are caused by a variety of fungal agents, which generally possess a limited invasiveness ${ }^{[1]}$. When producing a clinically apparent disease, the mycosis generally manifests as polymorphic, nodular or vegetative-verrucous lesions Fig. 1. Among the diseases caused by different etiological agents of fungal origin, chromoblastomycosis and Jorge Lobo's disease assume a particular importance in the Brazilian Amazon region due to their incidence related to the characteristics of the regional environment $t^{[1,2,3,4]}$.

Jorge Lobo's disease, or lacaziosis, is a chronic mycosis caused by Lacazia $\operatorname{loboi}^{[5,6]}$, which is characterized by keloidal cutaneous lesions mainly located in the auricle and lower limbs of humans ${ }^{[2,3]}$. Pecher (1988) suggested the presence of cellular immunodeficiency in patients with Jorge Lobo's disease, but a chronic inflammatory response accompanied by granuloma formation in which giant cells phagocytose a large number of fungi are observed in the lesions ${ }^{[7]}$. Lesion progression is slow, with new lesions developing in contiguity or appearing through the lymphatic route. Cases of Jorge Lobo's disease have been described in various Latin American countries, but the disease assumes great importance in the Brazilian Amazon region where the largest numbers of these cases are found $\mathrm{d}^{[1,2,3]}$.

Chromoblastomycosis is a mycosis that affects the skin and subcutaneous tissue and is characterized by the appearance of papular-desquamative lesions that slowly progress to polymorphic lesions ${ }^{[7]}$. The symptoms are discrete. Nodules, papules or ulcerations present separately or in groups may ulcerate, discharging a caseous secretion and leading to the emergence of verruciform masses with a cauliflower-like appearance $^{[4]}$. The lesions are generally found in

Corresponding Author: Juarez A.S. Quaresma, Tropical Medicine Unit, Federal do Para University, Av. Generalissimo eodoro 92, Umarizal, Belem, PA, Brazil, 66055-420 


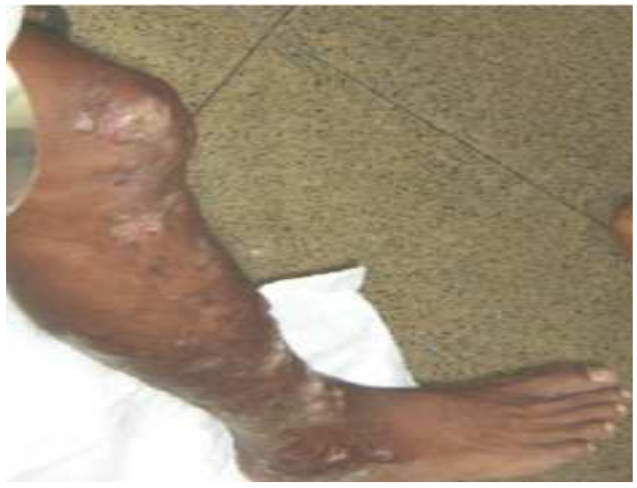

(A)

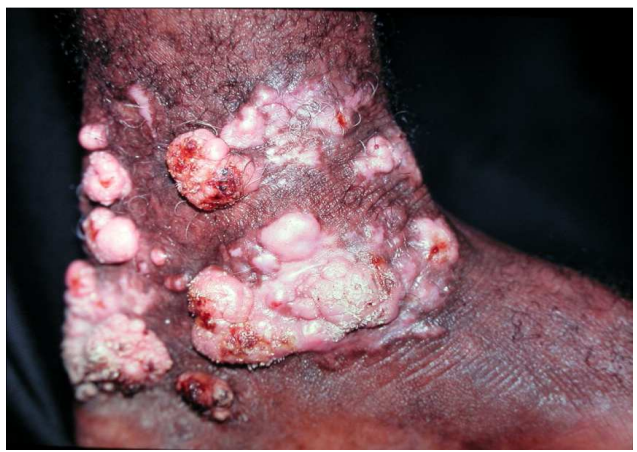

(B)

Fig. 1: (A): Nodular and vegetative-verrucous lesions in right leg, distributed of the knee to the ankle, in previous and external face. (B):Parakeloidal lesions in the leg

exposed areas and are almost always unilateral, being more frequent on the lower limbs. Muriform cells are observed upon microscopic analysis of pus or tissues $^{[4,8]}$.

\section{CASE REPORT}

A 50-year-old man, a laborer, living in the Anajás city, Para, Brazil (rural Amazon region), was seen at the Evandro Chagas Institute's ambulatory. The patient presented with an 18-year history of papular lesions, verrucous plaques and parakeloidal nodular lesions extending from the knee to the ankle on the front side of the right leg, accompanied by pain and local pruritus (Fig. 1 A, B). The patient had been treated during irregular periods with home-made medication and injectable antibiotic. Histopathological analysis of a skin fragment obtained from a verrucous lesion revealed intense hyperplasia and hyperkeratosis of the epidermis, sometimes showing well-circumscribed areas of an intraepidermal suppurative content intermingled with

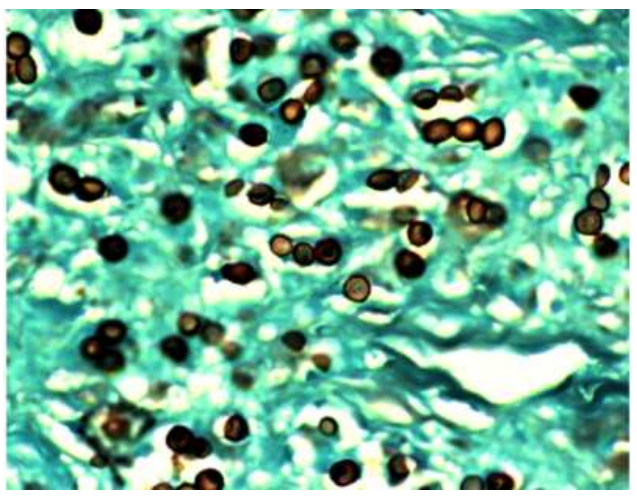

(A)

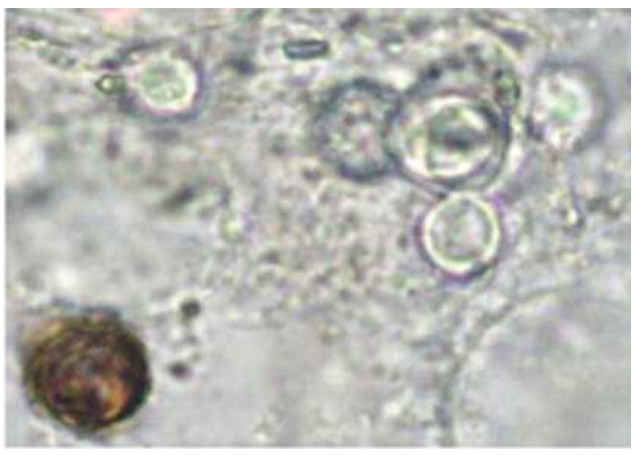

(B)

Fig. 2: (A): Histologic aspects of nodular lesions showing structures of Lacazia loboi in GomoriGrocott sections. (B): Direct micologic examination (solution of $\mathrm{KOH} 20 \%$ ) showing sclerotic cell in-group and structures presenting membrane of double contour, characteristics of Lacazia loboi

round and brownish structures. The papillary and reticular dermis was characterized by fibrosis and a granulomatous reaction consisting of lymphocytes, histiocytes and giant cells permeated by suppurative inflammatory foci and multiple round fungal structures mainly arranged amidst a neutrophilic inflammatory infiltrate.

The skin fragment obtained from a keloidal lesion showed atrophy and rectification of the epidermis, which was separated from the underlying dermis by a clearly visible band (Grenz zone). A granulomatous infiltrate extended into the reticular and deep dermis in a disperse manner. The infiltrate mainly consisted of macrophages and giant cells, which frequently contained intracytoplasmic fungal structures present separately or in groups. Dermal fibrosis was also observed. 
The clinical material collected from the lesion was submitted to direct mycological examination using $20 \%$ $\mathrm{KOH}$ solution and lactophenol cotton blue, which revealed the presence of yeast-like cells with a double membrane arranged in groups, a characteristic of Lacazia loboi, as well as muriform cells characteristic of chromoblastomycosis. Culture of the specimen on Mycosel agar and microculture on potato agar showed the typical morphology of Cladophialophora sp (Fig. 2 A, B).

\section{RESULT AND DISCUSSION}

Cutaneous infections caused by fungi of limited invasiveness are generally characterized by a chronic and protracted evolution ${ }^{[1]}$. Most patients originating from the different states that comprise the Brazilian Amazon region present evolutive symptoms of various years, including multiple lesions generally resulting from reinfections, autoinoculation and, although rare, lymphatic and hematogenous dissemination. Although most cases of chromoblastomycosis that occur in the Americas are caused by Fonsecaea pedrosoi, in the present case the agent involved was Cladophialophora sp., which is normally found as the causative agent of the disease in Australia. The location and aspect of the lesions, both in lacaziosis and chromoblastomycosis, were compatible with the pattern most commonly described in the literature, i.e., keloidal lesions and verrucous plaques predominantly located on the lower limbs.

The association of these two fungi causing lesions and inducing long-term disease may indicate a possible similarity in the characteristics of the habitat of these agents in the Amazon region. The relationship of the Amazonian population with rivers, forests and land cultivation led us to believe that these fungi are probably transmitted through water, soil and vegetation and inoculated into the dermis through trauma, initiating a long period of infection until the appearance of the first symptoms after some years. Some of these data are confirmed by the report of infection of dolphins with Lacazia loboi ${ }^{[9]}$, demonstrating the close ecological relationship of this fungus with water and consequently with the environment of the Amazon region. Finally, although the manifestations of chromoblastomycosis do not seem to be related to any type of immunodeficiency, some authors believe that the genesis of symptoms of Jorge Lobo's disease is associated with cellular immune deficiencies. Some of our studies have indicated an intense immunoexpression of TGF- $\beta$, an eminently immunosuppressive cytokine, in macrophages of cutaneous lesions in lacaziosis. On the other hand, no clinical changes or modifications in the histological pattern of the lesions have been observed in a patient with Jorge Lobo's disease co-infected with HIV/AIDS ${ }^{[10]}$. However, further studies are necessary to determine a possible correlation between disease susceptibility and the immune system's capacity to respond to the fungus.

Finally, the extensive forest areas and the exuberant hydrographical basin, together with the hot and humid climate, make the Amazon region an ideal environment for the maintenance and viability of important fungal agents that are potentially pathogenic to man. These factors, together with the intimate relationship between man and the environment, favor the occurrence of fungal diseases and, in view of the long distances from urban centers, contribute to the appearance of exuberant and sometimes atypical clinical forms, as well as uncommon associations. Both chromoblastomycosis and lacaziosis show a high incidence in hot and humid regions and, although chromoblastomycosis presents a more cosmopolitan distribution compared to lacaziosis, both diseases are endemic in the Amazon region. The incidence of lacaziosis is especially high in the Brazilian Amazon region and in bordering countries, while cases of the human disease become rarer as the distance from these regions increases ${ }^{[11]}$.

The great challenge in the understanding and management of these subcutaneous mycoses is represented by the poorly effective therapeutic procedures $^{[13]}$. Surgical ablation of the lesions can contribute to relative improvement of the cases. However, both diseases are characterized by a good prognosis in terms of systemic dissemination or life risk, but the therapeutic response is inefficient. On the other hand, the therapeutic management of these mycoses involves the use of highly expensive drugs for long periods of time, emphasizing the importance of an early diagnosis and adequate measures to attend the most affected populations, which mainly consist of laborers and rural dwellers from the Amazon region. In addition, in view of the long distances from urban centers with specialized medical care and the low social and economic condition of these patients, most of them are diagnosed during an advanced clinical phase, a fact that makes the choice of successful therapeutic measures even more difficult.

\section{REFERENCES}

1. Pecher, S.A., 1994. Deep mycoses in Latin America. Med. Trop., 54: 411-415.

2. Rodriguez-Toro, G. 1993. Lobomycosis. Int. J. Dermatol., 32: 324-332 
3. Silva, D., C. Macedo, C. Oliveira, D. Unger, 1996. Jorge Lobo's disease simulating gummy form: A rare case. Ann. Bras. Dermatol., 71: 211-213.

4. Lupi, O., S.K. Tyring, M.R. McGinnis, 2005. Tropical dermatology: Fungal tropical diseases. J. Am. Acad. Dermatol., 53: 931-951.

5. Taborda, P.R., V.A. Tabordam, M.R. McGinnis 1999. Lacazia loboi gen. nov., comb. nov., the Etiologic Agent of Lobomycosis. J. Clin. Microbiol., 37: 2031-2033.

6. Vilela, R., L. Mendonza, O.S. Rosa, A.F. Belone, S. Madeira, D.V. Opromolla, M.A. de Resende. 2005. Molecular model for studying the uncultivated fungal pathogen Lacazia loboi. J. Clin. Microbiol., 43: 3657- 3661.

7. Pecher, S.A., J. Funchs, 1988. Cellular immunity in lobomycosis (keliodal blastomycosis). Allergol. Immunol., 16: 413-415.

8. Palmeira, V.F., L.F. Kneipp, C.S. Alviano, A.L. Santos, 2006. The major chromoblastomycosis fungal pathogen, Fonsecaea pedrosoi, extracellularly releases proteolytic enzymes whose expression is modulated by culture medium composition: implications on the fungal development and cleavage of key's host structures. FEMS Immunol. Med. Microbiol., 46: 21-29.
9. Reif, J.S., M.S. Mazzoil, S.D. McCulloch, R.A. Varela, J.D. Goldstein, P.A. Fair, G.D. Bossart, 2006. Lobomycosis in Atlantic bottlenose dolphins from the Indian River Lagoon, Florida. J Am Vet Med Assoc. 2006; 228:104-108.

10. Xavier, M.B., M.M.R. Ferreira, J.A.S. Quaresma, A.C. de Brito, 2006. Hiv and Lacaziosis, Brazil. Emerg. Infect. Dis., 12: 526-527.

11. Elsayed, S., S.M. Kuhn, D. Barber, D.L. Church, S. Adams, R. Kasper, 2005. Human case of lobomycosis. Emerg. Infect. Dis., 10: 715-718.

12. Burns, R.A., J.S. Roy, C. Woods, A.A. Padhye D.W. Warnock, 2000. Report of the first human case of lobomycosis in the United States. J. Clin. Microbiol., 38: 1283-1285.

13. Restrepo, A., 1994. Treatment of Tropical mycosis. J. Am. Acad. Dermatol., 31: S91-110. 